\title{
ON TENSORS OF FACTORIZABLE QUANTUM CHANNELS WITH THE COMPLETELY DEPOLARIZING CHANNEL
}

\author{
Yuki Ueda
}

\begin{abstract}
In this paper, we obtain results for factorizability of quantum channels. Firstly, we prove that if a tensor $T \otimes S_{k}$ of a quantum channel $T$ on $M_{n}(\mathbb{C})$ with the completely depolarizing channel $S_{k}$ is written as a convex combination of automorphisms on the matrix algebra $M_{n}(\mathbb{C}) \otimes M_{k}(\mathbb{C})$ with rational coefficients, then the quantum channel $T$ has an exact factorization through some matrix algebra with the normalized trace. Next, we prove that if a quantum channel has an exact factorization through a finite dimensional von Neumann algebra with a convex combination of normal faithful tracial states with rational coefficients, then it also has an exact factorization through some matrix algebra with the normalized trace.
\end{abstract}

keywords: Markov maps, factorizable quantum channels, free group von Neumann algebras, completely depolarizing channels

\section{INTRODUCTION}

In [1], Anantharaman-Delaroche defined the class of factorizable Markov maps to study the noncommutative analogue of ergodic theory. After the works of [1], Haagerup and Musat proved in [3, Theorem 6.1] that every non-factorizable quantum channel on $M_{n}(\mathbb{C})$ $(n \geq 3)$ fails the asymptotic quantum Birkhoff conjecture which was raised by Smolin, Verstraete and Winter (see [7) as one of the most important problems in quantum information theory. In [3, 4], they also approached the Connes embedding problem by using factorizable quantum channels, in particular, tensors of factorizable quantum channels with the completely depolarizing channel. In this paper, we focus on the relation between the factorizability of quantum channels and the property of tensors of factorizable quantum channels with the completely depolarizing channel. Haagerup and Musat proved in 4 , Corollary 3.5] that if a quantum channel $T$ on $M_{n}(\mathbb{C})$ has an exact factorization through a tracial $W^{*}$-probability space $\left(M_{n}(\mathbb{C}) \otimes M_{k}(\mathbb{C}), \tau_{n} \otimes \tau_{k}\right)$, that is, there exists a unitary matrix $u$ in $M_{n}(\mathbb{C}) \otimes M_{k}(\mathbb{C})$ such that $T$ is written in the following form

$$
T x=\left(i d_{n} \otimes \tau_{k}\right)\left(u^{*}\left(x \otimes 1_{k}\right) u\right), \quad x \in M_{n}(\mathbb{C}),
$$

then $T \otimes S_{k} \in \operatorname{conv}\left(\operatorname{Aut}\left(M_{n}(\mathbb{C}) \otimes M_{k}(\mathbb{C})\right)\right)$. We raise the natural problem of whether the converse claim of the statement is true or not (see below).

Problem 1.1. Let $n$ be a positive integer and $T$ a quantum channel on $M_{n}(\mathbb{C})$. Is it true that the following properties are equivalent?

(1) There exists a positive integer $k$ such that $T$ has an exact factorization through 
$\left(M_{n}(\mathbb{C}) \otimes M_{k}(\mathbb{C}), \tau_{n} \otimes \tau_{k}\right)$.

(2) There exists a positive integer $k$ such that $T \otimes S_{k} \in \operatorname{conv}\left(\operatorname{Aut}\left(M_{n}(\mathbb{C}) \otimes M_{k}(\mathbb{C})\right)\right)$. The implication $(1) \Rightarrow(2)$ is true, but the implication $(2) \Rightarrow(1)$ is unknown.

We obtain a partial result for Problem 1.1 as follows.

Theorem 1.2. Let $T$ be a quantum channel on $M_{n}(\mathbb{C})$. If there exists a positive integer $k$ such that $T \otimes S_{k}=\sum_{i=1}^{d(k)} c_{i} \operatorname{ad}\left(u_{i}\right) \in \operatorname{conv}\left(\operatorname{Aut}\left(M_{n}(\mathbb{C}) \otimes M_{k}(\mathbb{C})\right)\right)$ for some positive integer $d(k)$, unitary matrices $u_{1}, \cdots, u_{d(k)} \in \mathcal{U}\left(M_{n}(\mathbb{C}) \otimes M_{k}(\mathbb{C})\right)$ and positive rational numbers $c_{1}, \cdots, c_{d(k)}$ with $\sum_{i=1}^{d(k)} c_{i}=1$, then there exists a positive integer $L$ such that $T$ has an exact factorization through $\left(M_{n}(\mathbb{C}) \otimes M_{L}(\mathbb{C}), \tau_{n} \otimes \tau_{L}\right)$.

Moreover we also raise the following problem for the quantum channels which have an exact factorization through a finite dimensional $W^{*}$-probability space (see below).

Problem 1.3. Let $T$ be a quantum channel on $M_{n}(\mathbb{C})$. Is it true that if there exists a finite dimensional $W^{*}$-probability space $(\mathcal{N}, \phi)$ (i.e. a pair of a finite dimensional von Neumann algebra $\mathcal{N}$ and a normal faithful state $\phi$ on $\mathcal{N})$ such that $T$ has an exact factorization through $\left(M_{n}(\mathbb{C}) \otimes \mathcal{N}, \tau_{n} \otimes \phi\right)$ then there exists a positive integer $k$ such that $T$ also has an exact factorization through $\left(M_{n}(\mathbb{C}) \otimes M_{k}(\mathbb{C}), \tau_{n} \otimes \tau_{k}\right)$ ?

Note that every finite dimensional von Neumann algebra is $*$-isomorphic to a direct sum of some matrix algebras. For this problem, we obtain the following partial result.

Theorem 1.4. Let $T$ be a quantum channel on $M_{n}(\mathbb{C})$. If for any $\alpha:=\left(\alpha_{1}, \cdots, \alpha_{d}\right) \in \mathbb{Q}_{+}^{d}$ with $\alpha_{1}+\cdots+\alpha_{d}=1$ there exist positive integers $k_{1}, \cdots, k_{d}$ such that $T$ has an exact factorization through $\left(M_{n}(\mathbb{C}) \otimes\left(M_{k_{1}}(\mathbb{C}) \oplus \cdots \oplus M_{k_{d}}(\mathbb{C})\right), \tau_{n} \otimes \tau_{\alpha}\right)$, where $\tau_{\alpha}$ is a normal faithful tracial state on $M_{k_{1}}(\mathbb{C}) \oplus \cdots \oplus M_{k_{d}}(\mathbb{C})$ defined by

$$
\tau_{\alpha}\left(x_{1}, \cdots, x_{d}\right):=\alpha_{1} \tau_{k_{1}}\left(x_{1}\right)+\cdots+\alpha_{d} \tau_{k_{d}}\left(x_{d}\right)
$$

for all $\left(x_{1}, \cdots, x_{d}\right) \in M_{k_{1}}(\mathbb{C}) \oplus \cdots \oplus M_{k_{d}}(\mathbb{C})$, then there exists a positive integer $k$ such that $T$ has an exact factorization through $\left(M_{n}(\mathbb{C}) \otimes M_{k}(\mathbb{C}), \tau_{n} \otimes \tau_{k}\right)$.

In section 2, we set notations and definitions in this paper. In section 3 , we recall and discuss for the concepts and basic properties of quantum channels, factorizable quantum channels and completely depolarizing channels. In section 4 and 5 , we prove that Theorem 1.2 and Theorem 1.4 hold, respectively.

\section{Notations and Definitions}

In this paper, we use the following notations:

- $\mathbb{N}:=\{1,2,3, \cdots\}$ and $\mathbb{Q}_{+}$is the set of all positive rational numbers.

- $M_{n}(\mathbb{C})$ is the set of all $n \times n$ matrices with complex entries.

- $\mathcal{U}(n)$ is the set of all $n \times n$ unitary matrices with complex entries.

- $\tau_{n}$ is the normalized trace on $M_{n}(\mathbb{C})$, i.e. $\tau_{n}\left(\left(x_{i j}\right)_{1 \leq i, j \leq n}\right):=\frac{x_{11}+\cdots x_{n n}}{n}$. 
- $i d_{n}$ is the identity map on $M_{n}(\mathbb{C})$.

- Define $\operatorname{ad}(u)(x):=u^{*} x u$ for all $x \in M_{n}(\mathbb{C}), u \in \mathcal{U}(n)$.

- $\operatorname{conv}\left(\operatorname{Aut}\left(M_{n}(\mathbb{C})\right)\right)$ is the convex hull of the set $\operatorname{Aut}\left(M_{n}(\mathbb{C})\right):=\{\operatorname{ad}(u): u \in \mathcal{U}(n)\}$.

- $1_{\mathcal{M}}$ is the unit in von Neumann algebra $\mathcal{M}$, in particular, $1_{n}:=1_{M_{n}(\mathbb{C})}$.

A pair $(\mathcal{M}, \phi)$ is called a $W^{*}$-probability space if $\mathcal{M}$ is a von Neumann algebra and $\phi$ is a normal faithful state on $\mathcal{M}$. In particular, we call $(\mathcal{M}, \phi)$ a tracial $W^{*}$-probability space when $\phi$ is tracial, that is $\phi(x y)=\phi(y x)$ for all $x, y \in \mathcal{M}$.

\section{BASIC PROPERTIES OF FACTORIZABLE QUANTUM CHANNELS}

In [1, Anantharaman-Delaroche considered factorizable Markov maps to prove a noncommutative analogue of Rota's theorem. We first recall the definition of Markov maps on a $W^{*}$-probability space. The concept is a noncommutative analogue of measure-preserving Markov operator on a probability space.

Definition 3.1. Let $(\mathcal{M}, \phi)$ and $(\mathcal{N}, \psi)$ be $W^{*}$-probability spaces. A linear map $T: \mathcal{M} \rightarrow$ $\mathcal{N}$ is called a $(\phi, \psi)$-Markov map if

(1) $T$ is completely positive,

(2) $T$ is unital,

(3) $T$ is $(\phi, \psi)$-preserving, i.e. $\psi \circ T=\phi$,

(4) $T \circ \sigma_{t}^{\phi}=\sigma_{t}^{\psi} \circ T$, where $\left\{\sigma_{t}^{\phi}\right\}_{t \in \mathbb{R}}$ denotes the automorphism group of the state $\phi$.

In particular, we call it $\phi$-Markov map when $(\mathcal{M}, \phi)=(\mathcal{N}, \psi)$.

If $(\mathcal{M}, \phi)=(\mathcal{N}, \psi)=\left(M_{n}(\mathbb{C}), \tau_{n}\right)$ in Definition 3.1, the fourth condition is removed since the operator $\sigma_{t}^{\tau}$ is trivial for any normal faithful tracial states $\tau$ on von Neumann algebras and $t \in \mathbb{R}$, so that a $\tau_{n}$-Markov map means a unital completely positive tracepreserving map (quantum channel) on $M_{n}(\mathbb{C})$. Denote by

$$
\mathcal{Q}(n):=\left\{T: M_{n}(\mathbb{C}) \rightarrow M_{n}(\mathbb{C}): T \text { is a quantum channel }\right\} .
$$

In [1, Definition 6.2], Anantharaman-Delaroche defined the class of factorizable Markov maps in the following sense.

Definition 3.2. A $(\phi, \psi)$-Markov map $T: \mathcal{M} \rightarrow \mathcal{N}$ is called factorizable if there exists a $W^{*}$-probability space $(\mathcal{L}, \chi)$ and $*$-monomorphisms $\alpha: \mathcal{M} \rightarrow \mathcal{L}$ and $\beta: \mathcal{N} \rightarrow \mathcal{L}$ such that $\alpha$ is $(\phi, \chi)$-Markov, $\beta$ is $(\psi, \chi)$-Markov and $T=\beta^{*} \circ \alpha$, where $\beta^{*}: \mathcal{L} \rightarrow \mathcal{M}$ is the adjoint of $\beta$ (see [3, Remark 1.2]).

The set of all factorizable $(\phi, \psi)$-Markov maps is closed under composition, the adjoint operation, taking convex combinations and $w^{*}$-limits (See [6, Proposition 2]). Haagerup and Musat proved in [3, Theorem 2.2] the following statement for the class of factorizable quantum channels.

Proposition 3.3. Consider $T \in \mathcal{Q}(n)$. Then the following properties are equivalent:

(1) $T$ is factorizable,

(2) There exists a tracial $W^{*}$-probability space $(\mathcal{M}, \phi)$ and a unitary $u$ in $M_{n}(\mathbb{C}) \otimes \mathcal{M}$ such that

$$
T x=\left(i d_{n} \otimes \phi\right)\left(u^{*}\left(x \otimes 1_{\mathcal{M}}\right) u\right), \quad x \in M_{n}(\mathbb{C}) .
$$


In this case, we say that $T$ has an exact factorization through $\left(M_{n}(\mathbb{C}) \otimes \mathcal{M}, \tau_{n} \otimes \phi\right)$. A factorization of quantum channels is not unique. We have two examples of a factorization of quantum channels. Firstly we show the following statement.

Lemma 3.4. If $T \in \mathcal{Q}(n)$ has an exact factorization through a tracial $W^{*}$-probability space $\left(M_{n}(\mathbb{C}) \otimes \mathcal{M}, \tau_{n} \otimes \phi\right)$ and there exist a tracial $W^{*}$-probability space $(\mathcal{N}, \psi)$ and a $(\phi, \psi)$-Markov ${ }^{*}$-homomorphism $S:(\mathcal{M}, \phi) \rightarrow(\mathcal{N}, \psi)$, then $T$ also has an exact factorization through $\left(M_{n}(\mathbb{C}) \otimes \mathcal{N}, \tau_{n} \otimes \psi\right)$.

Proof. Since $T$ has an exact factorization through $\left(M_{n}(\mathbb{C}) \otimes \mathcal{M}, \tau_{n} \otimes \phi\right)$, there exists a unitary $u \in M_{n}(\mathbb{C}) \otimes \mathcal{M}$ such that

$$
T x=\left(i d_{n} \otimes \phi\right)\left(u^{*}\left(x \otimes 1_{\mathcal{M}}\right) u\right), \quad x \in M_{n}(\mathbb{C}) .
$$

Since $S$ is a ${ }^{*}$-homomorphism, $\left(i d_{n} \otimes S\right)(u)$ is a unitary in $M_{n}(\mathbb{C}) \otimes \mathcal{N}$. Hence,

$$
\begin{aligned}
\left(i d_{n} \otimes \psi\right)\left(\left(i d_{n} \otimes S\right)(u)^{*}\left(x \otimes 1_{\mathcal{N}}\right)\left(i d_{n} \otimes S\right)(u)\right) & =\left(i d_{n} \otimes \psi\right)\left(i d_{n} \otimes S\right)\left(u^{*}\left(x \otimes 1_{\mathcal{M}}\right) u\right) \\
& =\left(i d_{n} \otimes \phi\right)\left(u^{*}\left(x \otimes 1_{\mathcal{M}}\right) u\right)=T x,
\end{aligned}
$$

for all $x \in M_{n}(\mathbb{C})$. Therefore $T$ has an exact factorization through $\left(M_{n}(\mathbb{C}) \otimes \mathcal{N}, \tau_{n} \otimes \psi\right)$.

As the second example, we consider a linear map $T$ defined by

$$
T x=\sum_{i=1}^{2} a_{i}^{*} x a_{i}, \quad x \in M_{2}(\mathbb{C}),
$$

where

$$
a_{1}:=\left(\begin{array}{cc}
1 & 0 \\
0 & 0
\end{array}\right), \quad a_{2}:=\left(\begin{array}{cc}
0 & 0 \\
0 & 1
\end{array}\right),
$$

has an exact factorization through $M_{2}(\mathbb{C}) \otimes \mathcal{L F}_{2}$. It is clear that $\sum_{i=1}^{2} a_{i}^{*} a_{i}=\sum_{i=1}^{2} a_{i} a_{i}^{*}=$ $1_{2}$. Hence $T$ is a quantum channel on $M_{2}(\mathbb{C})$ by [2]. Let $g_{1}, g_{2}$ be generators of the free group $\mathbb{F}_{2}$ of degree 2 and set $u:=\sum_{i=1}^{2} a_{i} \otimes \lambda_{g_{i}} \in \mathcal{U}\left(M_{2}(\mathbb{C}) \otimes \mathcal{L F}_{2}\right)$, where $\lambda_{g}$ is the left representation of $g \in \mathbb{F}_{2}$, that is,

$$
\lambda_{g}(f)(h):=f\left(g^{-1} h\right), \quad f \in l^{2} \mathbb{F}_{2}, \quad g, h \in \mathbb{F}_{2},
$$

and $\mathcal{L F}_{2}$ is the free group von Neumann algebra. For all $x \in M_{2}(\mathbb{C})$,

$$
\left(i d_{2} \otimes \tau_{\mathcal{L F}_{2}}\right)\left(u^{*}\left(x \otimes 1_{\mathcal{L F}_{2}}\right) u\right)=\sum_{i, j=1}^{2} \tau_{\mathcal{L F}_{2}}\left(\lambda_{g_{i}}^{*} \lambda_{g_{j}}\right) a_{i}^{*} x a_{j}=\sum_{i, j=1}^{2} \delta_{i j} a_{i}^{*} x a_{j}=T x,
$$

where

$$
\tau_{\mathcal{L F}_{2}}(\lambda):=<\lambda \delta_{e}, \delta_{e}>_{l^{2} \mathbb{F}_{2}}, \quad \lambda \in \mathcal{L F}_{2},
$$

and $e \in \mathbb{F}_{2}$ is the unit of $\mathbb{F}_{2}$. Therefore $T$ has an exact factorization through $\left(M_{2}(\mathbb{C}) \otimes\right.$ $\left.\mathcal{L F}_{2}, \tau_{2} \otimes \tau_{\mathcal{L F}_{2}}\right)$. On the other hand, $T$ also has an exact factorization through $\left(M_{2}(\mathbb{C}) \otimes\right.$ $\left.M_{4}(\mathbb{C}), \tau_{2} \otimes \tau_{4}\right)$ since $a_{1}, a_{2} \in M_{2}(\mathbb{C})$ are self-adjoint, $a_{1}^{2}+a_{2}^{2}=1_{2}$ and $a_{1} a_{2}=a_{2} a_{1}$ and therefore we can apply [3, Corollary 2.5] to the quantum channel T. Similarly we have the following statement. 
Proposition 3.5. Consider $d \geq 2$. Let $T$ be a quantum channel on $M_{d}(\mathbb{C})$ defined by

$$
T x:=\sum_{i=1}^{d} E_{i i}^{*} x E_{i i}, \quad x \in M_{d}(\mathbb{C}),
$$

where $\left\{E_{i j}\right\}_{1 \leq i, j \leq d}$ is the set of standard matrix units in $M_{d}(\mathbb{C})$. Then the following conditions hold.

(1) $T$ has an exact factorization through $\left(M_{d}(\mathbb{C}) \otimes \mathcal{L F} \mathbb{F}_{d}, \tau_{d} \otimes \tau_{\mathcal{L F}_{d}}\right)$.

(2) $T$ has an exact factorization through $\left(M_{d}(\mathbb{C}) \otimes M_{2^{d}}(\mathbb{C}), \tau_{d} \otimes \tau_{2^{d}}\right)$.

Denote by

$$
\mathcal{F}(n):=\{T \in \mathcal{Q}(n): T \text { is factorizable }\} .
$$

By the statements before Proposition 3.3 , we have that $\operatorname{conv}\left(\operatorname{Aut}\left(M_{n}(\mathbb{C})\right)\right) \subset \mathcal{F}(n)$ for all positive integers $n$. Haagerup and Musat found a quantum channel in $\mathcal{F}(n) \backslash \operatorname{conv}\left(\operatorname{Aut}\left(M_{n}(\mathbb{C})\right)\right)$ in [3, Example 3.3]. In particular, Kümmerer proved in [5] that $\operatorname{conv}\left(\operatorname{Aut}\left(M_{2}(\mathbb{C})\right)\right)=\mathcal{F}(2)$. Haagerup and Musat pointed out the important relations between the factorizable quantum channels and the Connes embedding problem in [3, 4].

Recall the completely depolarizing channels. Let $S_{k}: M_{n}(\mathbb{C}) \rightarrow M_{n}(\mathbb{C})$ be a linear map defined by

$$
S_{k}(x):=\tau_{k}(x) 1_{k}, \quad x \in M_{k}(\mathbb{C}) .
$$

The map $S_{k}$ is called the completely depolarizing channel on $M_{n}(\mathbb{C})$. Note that $S_{k}$ is in $\operatorname{conv}\left(\operatorname{Aut}\left(M_{k}(\mathbb{C})\right)\right)$ and therefore it is a factorizable quantum channel on $M_{n}(\mathbb{C})$. It is clear that $S_{k} \otimes S_{l}=S_{k l}$ for all $k, l \in \mathbb{N}$. It is very important to understand tensors $T \otimes S_{k}$ of a quantum channel $T$ with the completely depolarizing channel $S_{k}$ to know what $T$ has an exact factorization through some $W^{*}$-probability space. By [3, Corollary 2.5], Haagerup and Musat found a quantum channel $T \in \mathcal{F}(n) \backslash \operatorname{conv}\left(\operatorname{Aut}\left(M_{n}(\mathbb{C})\right)\right)(n \geq 3)$ such that it has an exact factorization through $\left(M_{n}(\mathbb{C}) \otimes M_{2^{d}}(\mathbb{C}), \tau_{n} \otimes \tau_{2^{d}}\right)$ for some $d \geq 3$. By 4 , Corollary 3.5], we have that $T \otimes S_{2^{d}} \in \operatorname{conv}\left(\operatorname{Aut}\left(M_{n}(\mathbb{C}) \otimes M_{2^{d}}(\mathbb{C})\right)\right)$.

\section{Proof of Theorem 1.2}

We prove that Theorem 1.2 holds. We first introduce the following sets.

$$
\begin{aligned}
& \mathcal{I}_{n}:=\left\{\begin{array}{l|l}
T \in \mathcal{F}(n) & \begin{array}{l}
\exists k \in \mathbb{N} \text { s.t. } T \otimes S_{k}=\sum_{i=1}^{d(k)} c_{i} \operatorname{ad}\left(u_{i}\right) \\
\text { for some positive integer } d(k), \\
c_{1}, \cdots, c_{d(k)} \in \mathbb{Q}_{+} \text {with } \sum_{i=1}^{d(k)} c_{i}=1, \\
\text { and } u_{1}, \cdots, u_{d(k)} \in \mathcal{U}\left(M_{n}(\mathbb{C}) \otimes M_{k}(\mathbb{C})\right)
\end{array}
\end{array}\right\}, \\
& \mathcal{J}_{n}:=\left\{\begin{array}{l|l}
T \in \mathcal{F}(n) & \begin{array}{l}
\exists k \in \mathbb{N} \text { s.t. } T \text { has an exact factorization through } \\
\left(M_{n}(\mathbb{C}) \otimes M_{k}(\mathbb{C}), \tau_{n} \otimes \tau_{k}\right)
\end{array}
\end{array}\right\} .
\end{aligned}
$$

By using these notations, we can rewrite as the statement of Theorem 1.2 ,

Theorem 4.1. Let $n \in \mathbb{N}$. Then $\mathcal{I}_{n} \subset \mathcal{J}_{n}$ holds. 
Proof. Suppose that $n \in \mathbb{N}$. If $T \in \mathcal{I}_{n}$, then there is a positive integer $k>0$ such that $T \otimes S_{k}(z)=\sum_{i=1}^{d(k)} c_{i} u_{i}^{*} z u_{i}$, for all $z \in M_{n}(\mathbb{C}) \otimes M_{k}(\mathbb{C})$ for some positive integer $d(k)>0$, unitaries $u_{1}, \cdots, u_{d(k)} \in M_{n}(\mathbb{C}) \otimes M_{k}(\mathbb{C})$, and positive rational numbers $c_{1}, \cdots, c_{d(k)}>0$ with $\sum_{i=1}^{d(k)} c_{i}=1$. Therefore

$$
T x=\left(i d_{n} \otimes \tau_{k}\right)\left(T \otimes S_{k}\right)\left(x \otimes 1_{k}\right)=\sum_{i=1}^{d(k)} c_{i}\left(i d_{n} \otimes \tau_{k}\right)\left(u_{i}^{*}\left(x \otimes 1_{k}\right) u_{i}\right), \quad x \in M_{n}(\mathbb{C}) .
$$

Since $c_{i}$ is a rational number for each $1 \leq i \leq d(k)$, we suppose that

$$
c_{i}=\frac{l_{i}}{L_{i}}, \quad 1 \leq i \leq d(k),
$$

where $l_{i}$ and $L_{i}$ are relatively prime positive numbers for each $i$. Let $L$ be the least common multiple of $L_{1}, L_{2}, \cdots, L_{d(k)}$. Then we can rewrite as:

$$
c_{i}=\frac{l_{i} \times \frac{L}{L_{i}}}{L}, \quad 1 \leq i \leq d(k)
$$

and rewrite as $C_{i}:=l_{i} \times \frac{L}{L_{i}} \in \mathbb{N}$ for each $i$. Note that $C_{1}+C_{2}+\cdots+C_{d(k)}=L$.

Then we set

$$
U:=\operatorname{diag}(\overbrace{u_{1}, \cdots, u_{1}}^{C_{1}}, \overbrace{u_{2} \cdots, u_{2}}^{C_{2}}, \cdots, \overbrace{u_{d(k)}, \cdots, u_{d(k)}}^{C_{d(k)}}) \in M_{n}(\mathbb{C}) \otimes M_{k}(\mathbb{C}) \otimes M_{L}(\mathbb{C}) .
$$

Clearly the block matrix $U$ is a unitary in $M_{n}(\mathbb{C}) \otimes M_{k}(\mathbb{C}) \otimes M_{L}(\mathbb{C})$, and we have that $\left(i d_{n} \otimes \tau_{k} \otimes \tau_{L}\right)\left(U^{*}\left(x \otimes 1_{k} \otimes 1_{L}\right) U\right)$

$$
\begin{aligned}
& =\left(i d_{n} \otimes \tau_{k} \otimes \tau_{L}\right)\left(\begin{array}{c}
\operatorname{diag}(\overbrace{u_{1}^{*}\left(x \otimes 1_{k}\right) u_{1}, \cdots, u_{1}^{*}\left(x \otimes 1_{k}\right) u_{1}}^{C_{1}}, \cdots \\
\cdots, \overbrace{u_{d(k)}^{*}\left(x \otimes 1_{l}\right) u_{d(k)}, \cdots, u_{d(k)}^{*}\left(x \otimes 1_{k}\right) u_{d(k)}}^{C_{d(k)}})
\end{array}\right) \\
& =\left(i d_{n} \otimes \tau_{k} \otimes \tau_{L}\right)\left(\begin{array}{c}
\sum_{i=1}^{C_{1}} u_{1}^{*}\left(x \otimes 1_{k}\right) u_{1} \otimes E_{i i}+\cdots \\
\cdots+\sum_{i=1}^{C_{d(k)}} u_{d(k)}^{*}\left(x \otimes 1_{k}\right) u_{d(k)} \otimes E_{L-c_{d(k)}+i, L-c_{d(k)}+i}
\end{array}\right) \\
& =\begin{array}{l}
\sum_{i=1}^{C_{1}} \tau_{L}\left(E_{i i}\right)\left(i d_{n} \otimes \tau_{k}\right)\left(u_{1}^{*}\left(x \otimes 1_{k}\right) u_{1}\right)+\cdots \\
\cdots+\sum_{i=1}^{C_{d(k)}} \tau_{L}\left(E_{L-c_{d(k)}+i, L-c_{d(k)}+i}\right)\left(i d_{n} \otimes \tau_{k}\right)\left(u_{d(k)}^{*}\left(x \otimes 1_{k}\right) u_{d(k)}\right)
\end{array} \\
& =\sum_{i=1}^{d(k)} \frac{C_{i}}{L}\left(i d_{n} \otimes \tau_{k}\right)\left(u_{i}^{*}\left(x \otimes 1_{k}\right) u_{i}\right) \\
& =\sum_{i=1}^{d(k)} c_{i}\left(i d_{n} \otimes \tau_{k}\right)\left(u_{i}^{*}\left(x \otimes 1_{k}\right) u_{i}\right)=T x,
\end{aligned}
$$

for all $x \in M_{n}(\mathbb{C})$, where $\left\{E_{i j}\right\}_{1 \leq i, j \leq L}$ is the set of standard matrix units in $M_{L}(\mathbb{C})$. Therefore $T$ has an exact factorization through $\left(M_{n}(\mathbb{C}) \otimes\left(M_{k}(\mathbb{C}) \otimes M_{L}(\mathbb{C})\right), \tau_{n} \otimes\left(\tau_{k} \otimes \tau_{L}\right)\right)$. Hence we have the inclusion $\mathcal{I}_{n} \subset \mathcal{J}_{n}$, for each positive integer $n \in \mathbb{N}$. Thus the proof is complete. 


\section{Proof of Theorem 1.4}

We prove that Theorem 1.4 holds. We also denote the following set.

$$
\mathcal{K}_{n}:=\left\{T \in \mathcal{F}(n): \exists k \in \mathbb{N} \text { s.t. } T \otimes S_{k} \in \operatorname{conv}\left(\operatorname{Aut}\left(M_{n}(\mathbb{C}) \otimes M_{k}(\mathbb{C})\right)\right)\right\} .
$$

Clearly $\operatorname{conv}\left(\operatorname{Aut}\left(M_{n}(\mathbb{C})\right)\right) \subset \mathcal{K}_{n}$. The statement before the condition (1.1) in section 1 or [4. Corollary] implies that $\mathcal{J}_{n} \subset \mathcal{K}_{n}$ holds for each positive integer $n$. By the last statements in section 3 , the set $\mathcal{K}_{n} \backslash \operatorname{conv}\left(\operatorname{Aut}\left(M_{n}(\mathbb{C})\right)\right)$ ) is not empty for all $n \geq 3$. We can rewrite as the statement of Theorem 1.4 by the new notations in sections 4 and 5 .

Theorem 5.1. Let $n \in \mathbb{N}$. If for any $\alpha:=\left(\alpha_{1}, \cdots, \alpha_{d}\right) \in \mathbb{Q}_{+}^{d}$ with $\alpha_{1}+\cdots+\alpha_{d}=1$ there exist some positive integers $k_{1}, \cdots, k_{d}$ such that $T \in \mathcal{F}(n)$ has an exact factorization through $\left(M_{n}(\mathbb{C}) \otimes\left(M_{k_{1}}(\mathbb{C}) \oplus \cdots \oplus M_{k_{d}}(\mathbb{C})\right), \tau_{n} \otimes \tau_{\alpha}\right)$, where $\tau_{\alpha}$ is defined by (1.2), then $T \in \mathcal{J}_{n}$, and therefore $T \in \mathcal{K}_{n}$.

Proof. (Step 1) Suppose that $\alpha:=\left(\alpha_{1}, \cdots, \alpha_{d}\right) \in \mathbb{Q}_{+}^{d}$ with $\alpha_{1}+\cdots+\alpha_{d}=1$ and there exist $k \in \mathbb{N}$ and the normal faithful tracial state $\tau_{\alpha}$ defined by

$$
\tau_{\alpha}\left(x_{1}, \cdots, x_{d}\right):=\alpha_{1} \tau_{k}\left(x_{1}\right)+\cdots+\alpha_{d} \tau_{k}\left(x_{d}\right), \quad\left(x_{1}, \cdots, x_{d}\right) \in \overbrace{M_{k}(\mathbb{C}) \oplus \cdots \oplus M_{k}(\mathbb{C})}^{d}
$$

such that $T$ has an exact factorization through $\left(M_{n}(\mathbb{C}) \otimes(\overbrace{M_{k}(\mathbb{C}) \oplus \cdots \oplus M_{k}(\mathbb{C})}^{d}), \tau_{n} \otimes \tau_{\alpha}\right)$. Since $\alpha_{1}, \cdots, \alpha_{d}$ are rational numbers, we can write as

$$
\alpha_{i}=\frac{l_{i}}{L}, \quad 1 \leq i \leq d,
$$

where $l_{i}$ and $L$ are positive integers and $l_{1}+\cdots+l_{d}=L$. We will define the following map:

$\phi: \overbrace{M_{k}(\mathbb{C}) \oplus \cdots \oplus M_{k}(\mathbb{C})}^{d} \rightarrow M_{k L}(\mathbb{C}), \quad\left(x_{1}, \cdots, x_{d}\right) \mapsto \operatorname{diag}(\overbrace{x_{1}, \cdots, x_{1}}^{l_{1}}, \cdots, \overbrace{x_{d}, \cdots, x_{d}}^{l_{d}})$

By the definition of $\phi$, it is easy to check that $\phi$ is a unital completely positive *homomorphism. We consider $x_{i}=\left(x_{s l}^{i}\right)_{1 \leq s, l \leq k} \in M_{k}(\mathbb{C})(1 \leq i \leq d)$. Then

$$
\tau_{k L} \circ \phi\left(x_{1}, \cdots, x_{d}\right)=\tau_{k L}\left(\operatorname{diag}(\overbrace{x_{1}, \cdots, x_{1}}^{l_{1}}, \cdots, \overbrace{x_{d}, \cdots, x_{d}}^{l_{d}})=\frac{1}{k L} \sum_{i=1}^{d} \sum_{j=1}^{k} l_{i} x_{j j}^{i} .\right.
$$

On the other hand,

$$
\tau_{\alpha}\left(x_{1}, \cdots, x_{d}\right)=\sum_{i=1}^{d} \alpha_{i} \tau_{k}\left(x_{i}\right)=\sum_{i=1}^{d} \frac{l_{i}}{L}\left(\frac{1}{k} \sum_{j=1}^{k} x_{j j}^{i}\right)=\frac{1}{k L} \sum_{i=1}^{d} \sum_{j=1}^{k} l_{i} x_{j j}^{i} .
$$

Therefore $\phi$ is a unital completely positive $\left(\tau_{\alpha}, \tau_{k L}\right)$-preserving $*$-homomorphism from $(\overbrace{M_{k}(\mathbb{C}) \oplus \cdots \oplus M_{k}(\mathbb{C})}^{d}, \tau_{\alpha})$ to $\left(M_{k L}(\mathbb{C}), \tau_{k L}\right)$. By Lemma 3.4, $T$ also has an exact factorization through $\left(M_{n}(\mathbb{C}) \otimes M_{k L}(\mathbb{C}), \tau_{n} \otimes \tau_{k L}\right)$. By [4, Corollary 3.5], $T \otimes S_{k L} \in \operatorname{conv}\left(\operatorname{Aut}\left(M_{n}(\mathbb{C}) \otimes\right.\right.$ 
$\left.\left.M_{k L}(\mathbb{C})\right)\right)$. Therefore $T \in \mathcal{J}_{n} \subset \mathcal{K}_{n}$.

(Step 2) Suppose that $\alpha:=\left(\alpha_{1}, \alpha_{2}\right) \in \mathbb{Q}_{+}^{2}$ with $\alpha_{1}+\alpha_{2}=1$ and assume that $T$ has an exact factorization through $\left(M_{n}(\mathbb{C}) \otimes\left(M_{k_{1}}(\mathbb{C}) \oplus M_{k_{2}}(\mathbb{C})\right), \tau_{n} \otimes \tau_{\alpha}\right)$ (In general, $\left.k_{1} \neq k_{2}\right)$, where $\tau_{\alpha}$ is a normal faithful tracial state on $M_{k_{1}}(\mathbb{C}) \otimes M_{k_{2}}(\mathbb{C})$ defined by

$$
\tau_{\alpha}(x, y):=\alpha_{1} \tau_{k_{1}}(x)+\alpha_{2} \tau_{k_{2}}(y), \quad(x, y) \in M_{k_{1}}(\mathbb{C}) \otimes M_{k_{2}}(\mathbb{C}) .
$$

We define the following map:

$\psi: M_{k_{1}}(\mathbb{C}) \oplus M_{k_{2}}(\mathbb{C}) \rightarrow M_{k_{1} k_{2}}(\mathbb{C}) \oplus M_{k_{1} k_{2}}(\mathbb{C}), \quad(x, y) \mapsto\left(\operatorname{diag}(\overbrace{x, \cdots, x}^{k_{2}}), \operatorname{diag}(\overbrace{y, \cdots, y}^{k_{1}})\right)$.

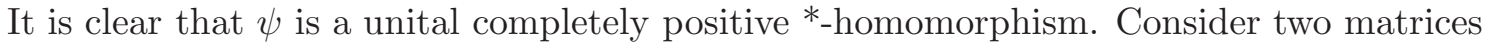
$x=\left(x_{i j}\right)_{1 \leq i, j \leq k_{1}} \in M_{k_{1}}(\mathbb{C})$ and $y=\left(y_{i j}\right)_{1 \leq i, j \leq k_{2}} \in M_{k_{2}}(\mathbb{C})$. Then

$$
\begin{aligned}
\left(\alpha_{1} \tau_{k_{1} k_{2}} \oplus \alpha_{2} \tau_{k_{1} k_{2}}\right) \circ \psi(x, y) & =\left(\alpha_{1} \tau_{k_{1} k_{2}} \oplus \alpha_{2} \tau_{k_{1} k_{2}}\right)\left(\operatorname{diag}(\overbrace{x, \cdots, x}^{k_{2}}), \operatorname{diag}(\overbrace{y, \cdots, y}^{k_{1}})\right) \\
& =\alpha_{1} \tau_{k_{1} k_{2}}\left(\operatorname{diag}(\overbrace{x, \cdots, x}^{k_{2}})\right)+\alpha_{2} \tau_{k_{1} k_{2}}\left(\operatorname{diag}(\overbrace{y, \cdots, y}^{k_{1}})\right) \\
& =\alpha_{1}\left(\frac{k_{2}}{k_{1} k_{2}} \sum_{i=1}^{k_{1}} x_{i i}\right)+\alpha_{2}\left(\frac{k_{1}}{k_{1} k_{2}} \sum_{j=1}^{k_{2}} y_{j j}\right) \\
& =\frac{\alpha_{1}}{k_{1}} \sum_{i=1}^{k_{1}} x_{i i}+\frac{\alpha_{2}}{k_{2}} \sum_{j=1}^{k_{2}} y_{j j} \\
& =\alpha_{1} \tau_{k_{1}}(x)+\alpha_{2} \tau_{k_{2}}(y)=\tau_{\alpha}(x, y),
\end{aligned}
$$

where $\alpha_{1} \tau_{k_{1} k_{2}} \oplus \alpha_{2} \tau_{k_{1} k_{2}}$ is a normal faithful tracial state on $M_{k_{1} k_{2}}(\mathbb{C}) \oplus M_{k_{1} k_{2}}(\mathbb{C})$ defined by

$$
\alpha_{1} \tau_{k_{1} k_{2}} \oplus \alpha_{2} \tau_{k_{1} k_{2}}(x, y):=\alpha_{1} \tau_{k_{1} k_{2}}(x)+\alpha_{2} \tau_{k_{1} k_{2}}(y), \quad(x, y) \in M_{k_{1} k_{2}}(\mathbb{C}) \oplus M_{k_{1} k_{2}}(\mathbb{C}) .
$$

Therefore $\psi$ is a unital completely positive $\left(\tau_{\alpha}, \alpha_{1} \tau_{k_{1} k_{2}} \oplus \alpha_{2} \tau_{k_{1} k_{2}}\right)$-preserving *-homomorphism. By using Lemma 3.4, $T$ has an exact factorization through $\left(M_{n}(\mathbb{C}) \otimes\left(M_{k_{1} k_{2}}(\mathbb{C}) \oplus M_{k_{1} k_{2}}(\mathbb{C})\right), \tau_{n} \otimes\right.$ $\left.\left(\alpha_{1} \tau_{k_{1} k_{2}} \oplus \alpha_{2} \tau_{k_{1} k_{2}}\right)\right)$. Denote by

$$
\alpha_{1}=\frac{l_{1}}{L}, \alpha_{2}=\frac{l_{2}}{L}, \quad l_{1}, l_{2}, L \in \mathbb{N} \text { with } l_{1}+l_{2}=L .
$$

By using the first step, $T$ has an exact factorization through $\left(M_{n}(\mathbb{C}) \otimes M_{k_{1} k_{2} L}(\mathbb{C}), \tau_{n} \otimes\right.$ $\tau_{k_{1} k_{2} L}$ ), and therefore $T \in \mathcal{J}_{n} \subset \mathcal{K}_{n}$ by [4, Corollary 3.5].

(Step 3) Assume that $\alpha:=\left(\alpha_{1}, \cdots, \alpha_{d}\right) \in \mathbb{Q}_{+}^{d}$ with $\alpha_{1}+\cdots+\alpha_{d}=1$ and there exist some positive integers $k_{1}, \cdots, k_{d}$ and a normal faithful tracial state $\tau_{\alpha}$ on $M_{k_{1}}(\mathbb{C}) \oplus \cdots \oplus M_{k_{d}}(\mathbb{C})$ such that $T$ has an exact factorization through $\left(M_{n}(\mathbb{C}) \otimes\left(M_{k_{1}}(\mathbb{C}) \oplus \cdots \oplus M_{k_{d}}(\mathbb{C})\right), \tau_{n} \otimes \tau_{\alpha}\right)$. By using repeatedly first step or second step, there exists positive integer $k$ such that $T$ also has an exact factorization through $\left(M_{n}(\mathbb{C}) \otimes M_{k}(\mathbb{C}), \tau_{n} \otimes \tau_{k}\right)$. Therefore $T \in \mathcal{J}_{n} \subset \mathcal{K}_{n}$ by [4, Corollary 3.5] again. 


\section{ACKNOWLEDGMENT}

This paper is a revised version of the master's thesis of the author. The author would like to express his heartly thanks to Professor Benoit Collins for carefully reading this paper and pointing out some inaccuracies. The author had a chance to visit Professor Magdalena Musat and Professor Mikael Rørdam (in Copenhagen university) during November-December, 2016 with the support of the Top Global University project for Kyoto University. The author would appreciate their hospitality when the author was staying at Copenhagen University. In particular, thanks to their important advices, the author could advance researches related to the sections 4 and 5 .

\section{REFERENCES}

[1] C. Anantharaman-Delaroche, On ergodic theorems for free group actions on noncommutative spaces, Probab. Theory Related Fields 135 (2006), no. 4, 520-546.

[2] M. D. Choi, Completely positive linear maps on complex matrices, Linear Algebra and Appl. 10, Issue 3, (1975), 285-290.

[3] U. Haagerup, M. Musat, Factorization and dilation problems for completely positive maps on von Neumann algebras. Comm. Math. Phys. 303 (2011), no. 2, 555-594.

[4] U. Haagerup, M. Musat, An asymptotic property of factorizable completely positive maps and the Connes embedding problem. Comm. Math. Phys. 338 (2015), no. 2, 721-752.

[5] B. Kümmerer, Markov dilations on the $2 \times 2$ matrices, In Operator algebras and their connections with topology and ergodic theory (Busteni, 1983), Lect. Notes Math. 1132, Berlin, Springer, 1985, 312-323.

[6] E. Ricard, A Markov dilation for self-adjoint Schur multipliers, Proc. Amer. Math. Soc. 136 (2008), no. 12, 4365-4372.

[7] J. A. Smolin, F. Verstraete, A. Winter, Entanglement of assistance and multipartite state distillation, Phys. Rev. A 72, 052317 (2005) 1-10.

Yuki Ueda

Department of Mathematics, Hokkaido University,

Kita 10, Nishi 8, Kita-Ku, Sapporo, Hokkaido, 060-0810, Japan

email:yuuki1114@math.sci.hokudai.ac.jp 